\title{
High-power fiber lasers for photocathode electron injectors
}

\author{
Zhi Zhao, ${ }_{1}^{1}$ Adam Bartnik, ${ }^{1}$ Frank W. Wise, ${ }^{2}$ Ivan V. Bazarov, ${ }^{1}$ and Bruce M. Dunham ${ }^{1}$ \\ ${ }^{1}$ Department of Physics, CLASSE, Cornell University, Ithaca, New York 14853, USA \\ ${ }^{2}$ School of Applied and Engineering Physics, Cornell University, Ithaca, New York 14853, USA
}

(Received 7 February 2014; published 5 May 2014)

\begin{abstract}
Many new applications for electron accelerators require high-brightness, high-average power beams, and most rely on photocathode-based electron injectors as a source of electrons. To achieve such a photoinjector, one requires both a high-power laser system to produce the high average current beam, and also a system at reduced repetition rate for electron beam diagnostics to verify high beam brightness. Here we report on two fiber laser systems designed to meet these specific needs, at $50 \mathrm{MHz}$ and $1.3 \mathrm{GHz}$ repetition rate, together with pulse pickers, second harmonic generation, spatiotemporal beam shaping, intensity feedback, and laser beam transport. The performance and flexibility of these laser systems have allowed us to demonstrate electron beam with both low emittance and high average current for the Cornell energy recovery linac.
\end{abstract}

DOI: 10.1103/PhysRevSTAB.17.053501

PACS numbers: 42.55.-f, 42.62.-b, 41.75.Jv

\section{INTRODUCTION}

Intense electron beams are key to a large number of scientific endeavors, including electron cooling of hadron beams, electron-positron colliders, secondary-particle beams such as photons and positrons, and new highgradient accelerators that use electron-driven plasmas. Many applications for high-intensity electron beams have arisen in recent years in high-energy physics, nuclear physics, and energy sciences. These concepts rely on high-intensity, linac-quality beams to provide or facilitate adequate flux for high luminosity at the interaction point. Recent designs for an electron-hadron collider at CERN (LHeC) [1], beam coolers for hadron beams at LHC and eRHIC [2], and a hard x-ray source at Cornell University [3] provide excellent examples.

Photoinjectors are used at the majority of highbrightness electron linacs today, due to their efficiency, timing structure flexibility and ability to produce high power, high brightness beams. Cornell University began research on a high brightness photoinjector as a driver for an x-ray light source in 2005, with the goal of delivering an electron beam with low emittance, up to $15 \mathrm{MeV}$ beam energy, and $100 \mathrm{~mA}$ average current at $1.3 \mathrm{GHz}$ bunch rate [4]. The requirements for the Cornell Energy Recovery Linac (ERL) photoinjector are listed in Table I.

These parameters set the requirements for the laser system needed to operate this photoinjector. Many innovations were necessary to successfully realize the reliable laser systems, which have been a key prerequisite for

Published by the American Physical Society under the terms of the Creative Commons Attribution 3.0 License. Further distribution of this work must maintain attribution to the author(s) and the published article's title, journal citation, and DOI. demonstration of high brightness beams from the Cornell photoinjector. These advances include: (1) nonlinear and thermal management to achieve $\sim 60 \mathrm{~W}$ green power at $1.3 \mathrm{GHz}$ repetition rate, as required for high current ERLs [5]; (2) birefringent crystal shaping of the laser [6] and electron temporal profile $[7,8]$ to reduce the effect of space charge; (3) laser transport design for good transverse pointing stability and transverse shaping to achieve low emittance [9]; (4) laser intensity feedback to suppress the noise spurs and deliver a reliable electronic beam for the further acceleration.

To determine the beam emittance, a separate laser system operating at $50 \mathrm{MHz}$ repetition ration rate is built, as these diagnostics are interceptive and cannot withstand full beam power. In order to reduce the average laser power and thus the beam current, a pulse picker could be used on the laser beam, reducing average power while maintaining the energy in each pulse (and therefore the same bunch charge in each electron bunch). This is still not sufficient for two reasons. First, a given pulse picker has finite rise and fall times. During those times, the laser will be producing pulses with varying amounts of energy, and the photoinjector will generate varying charge bunches. Since varying bunch charges would confuse the diagnostic process, the pulse picker should remain "on" for a long time

TABLE I. Requirements for the Cornell photoinjector.

\begin{tabular}{lc}
\hline \hline Parameter & Value \\
\hline Frequency & $1300 \mathrm{MHz}$ \\
Charge per bunch & $77 \mathrm{pC}$ \\
Average current & $100 \mathrm{~mA}$ \\
Normalized emittance & $\leq 0.3 \mu \mathrm{m}$ \\
Bunch duration & $2-3 \mathrm{ps} \mathrm{rms}$ \\
Beam energy & $4-15 \mathrm{MeV}$ \\
\hline \hline
\end{tabular}


TABLE II. Laser requirements for high power operation (diagnostic operation).

\begin{tabular}{lc}
\hline \hline Parameter & Value \\
\hline Min power at the cathode & $50 \mathrm{~W}(2 \mathrm{~W})$ \\
Wavelength & $520 \mathrm{~nm}$ \\
Pointing stability & $10 \mu \mathrm{m} \mathrm{rms}$ \\
Pulse length at the cathode & $20-30$ ps flattop \\
Transverse shape at the cathode & $1-3 \mathrm{~mm}$ diameter truncated \\
& Gaussian \\
Extinction ratio & $>10^{6}$ \\
Frequency & $1.3 \mathrm{GHz}(50 \mathrm{MHz})$ \\
\hline \hline
\end{tabular}

compared to the rise/fall time. Second, a pulse picker will also have a finite extinction ratio - that is, the ratio in power between allowed and disallowed pulses. One would want the average power contained in all of the "off" pulses to be small compared with the average power in the "on" pulses, to reduce unwanted background.

Both of those reasons set lower bounds to the duty factor one can use in a pulse picker. Practically, it becomes much simpler to design a second laser system with a reduced repetition rate, than it is to fight against the finite rise time and extinction ratio in a pulse picker. $50 \mathrm{MHz}$ is a convenient repetition rate, with a factor of 26 less average power, primarily because it allows a simple design for the laser oscillator.

A summary of the requirements for both high-power operation and diagnostic operation are shown in Table II. A minimum cathode quantum efficiency (QE) of $1 \%$ is assumed, and sets the minimum laser power required.

This paper is devoted to a detailed description of the $50 \mathrm{MHz}$ and $1.3 \mathrm{GHz}$ high-power fiber laser systems for the Cornell energy recovery linac (ERL) photoinjector. The high-power fiber amplifiers and second harmonic generation that delivers the necessary average power will be described first, followed by a number of the key ingredients necessary for efficient beam shaping, diagnostics, and control. These components include optical pulse pickers,

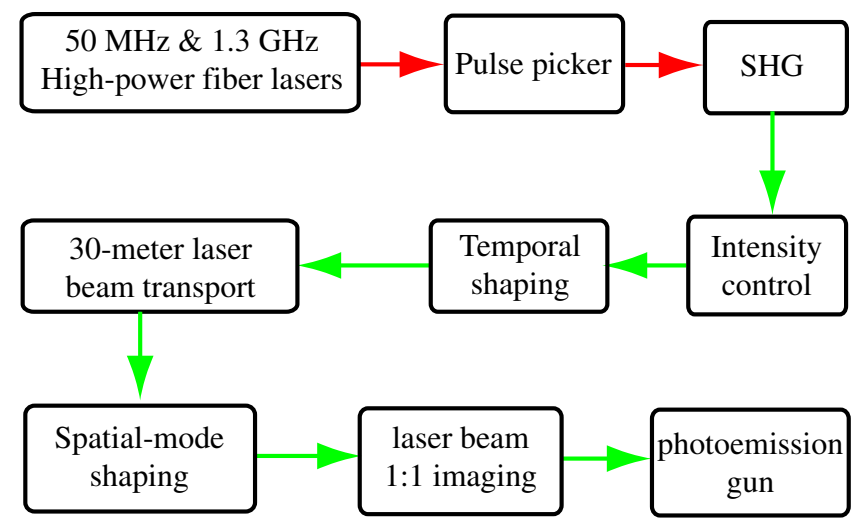

FIG. 1. Overview of the laser system for Cornell ERL photoinjector. light intensity control, average beam current stabilization, laser beam transport, and longitudinal and transverse laser beam shaping (see Fig. 1). Finally, we will briefly discuss the operation of the laser for low emittance and the high average current electron beams for the Cornell ERL photoinjector.

\section{50 MHz LASER SYSTEM AND CHARACTERIZATION}

The schematic of the $50 \mathrm{MHz}$ laser system is shown in Fig. 2. The Yb-doped fiber master oscillator power amplifier (MOPA) consists of a seed source, a preamp, and a main amplifier. The output pulses of the amplifier are steered through a Pockels cell (PC) and converted into green light through second harmonic generation (SHG). A similar system has been described in [10], but the entire amplifier system has since been redesigned to double the output power and improve the stability of optical pulses.

The seed source is a $\mathrm{Yb}$-fiber oscillator in a ring cavity (Fig. 3), operated in the soliton regime [11]. The cavity features three segments of fiber. The initial $70 \mathrm{~cm}$ of singlemode fiber (SMF) was chosen to achieve a repetition rate near $50 \mathrm{MHz}$, followed by $55 \mathrm{~cm}$ of gain fiber (Yb501, CorActive). Finally, $2 \mathrm{~m}$ of SMF allows enough nonlinear polarization evolution for stable, self-starting mode locking to occur even at the low pulse energies found in soliton oscillators. The three wave plates surrounding the fiber segments are used to mode lock the laser. Two 1200 lines/ $\mathrm{mm}$ gratings have an incident angle of 50 degrees and a separation of $21 \mathrm{~cm}$, for a net dispersion of negative $1.8 \mathrm{ps}^{2}$. The subsequent slit has a tunable width and is mounted on a translation stage. It is set to roughly $1 \mathrm{~mm}$ width, creating a $4 \mathrm{~nm}$ tunable spectral filter, translated to fix the central wavelength at $1040 \mathrm{~nm}$.

A small sigma-cavity segment follows the pickoff mirror, with a piezoelectric transducer mounted on a motorized translation stage. The piezo is used to lock the frequency to

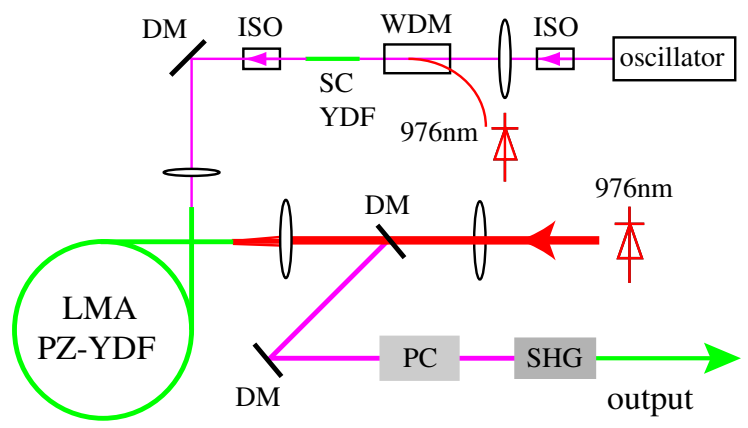

FIG. 2. Schematic of $50 \mathrm{MHz}$ laser system: LMA, large mode area; PZ-YDF, single-polarization Yb-doped fiber; WDM, wavelength division multiplexer; SC, single clad; ISO, optical isolator; DM, dichroic mirror; PC, Pockels cell; SHG, second harmonic generation. 


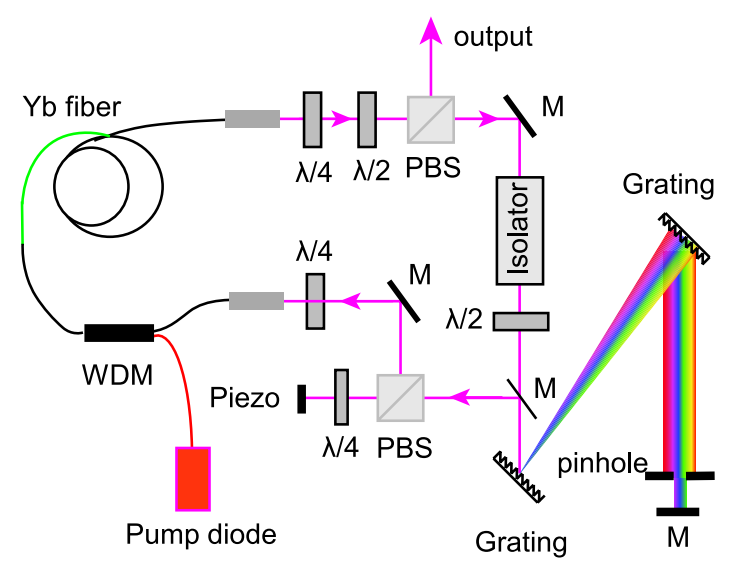

FIG. 3. Schematic of the $50 \mathrm{MHz}$ oscillator cavity: $\lambda / 2$, halfwave plate; $\lambda / 4$, quarter-wave plate; $\mathrm{PBS}$, polarization beam splitter; M, mirror; grating, 1200 lines per mm; WDM, wavelength division multiplexer; Yb fiber, 55-cm ytterbium-doped gain fiber.

an external $50 \mathrm{MHz}$ clock, and the translation stage is used to keep the piezo voltage in its optimum range.

In practice, the oscillator is mode locked by adjusting the three wave plates and the width of the slit until pulses are observed on a fast photodiode while looking for a broad soliton spectrum on an optical spectrum analyzer. After the laser is mode locked, the wave plates are adjusted further until the laser remains mode locked even if the angle is changed by \pm 5 degrees. If the settings are stable, the laser will self-start in a fraction of a second after briefly blocking the beam. By following this procedure, continuous operation on the order of many weeks is typical, with occasional adjustments mostly due to changes in temperature and humidity.

Improvements to the cavity design may improve stability further. The sigma-cavity arm was used so that the cavity would not change alignment as the piezo mirror moves. In practice, the cavity must be intentionally misaligned in this arm, or the mode locking is unstable. With perfect alignment, there is a small amount of back-reflection, determined by the extinction ratio of the polarization beam cube and the quality of the quarter-wave plate, and this back-reflection into the gain fiber creates a subcavity that competes for the gain in the fiber, making mode locking unstable. This defeats the purpose of the sigma arm, and a new design where the piezo is mounted at the end of the arm containing the gratings would work just as well, have fewer parts, and be easier to align.

The piezo also changes angle as a function of voltage. If the cavity is aligned for one voltage, then another voltage will have a slightly misaligned cavity. A better quality piezo should be used that is designed to have improved angle stability. In practice, the angle from the piezo is also adjusted once every few weeks, but rather than optimizing it for one voltage, the cavity is intentionally slightly misaligned for all voltages, so that the cavity output power is roughly constant with voltage. This helps to ensure longterm stability.

As opposed to the room containing the $1.3 \mathrm{GHz}$ laser, no effort has been made to keep the temperature or humidity stable for the $50 \mathrm{MHz}$ system. Typically the temperature will vary $\pm 0.5^{\circ} \mathrm{C}$, but may have drifts of $2{ }^{\circ} \mathrm{C}-3{ }^{\circ} \mathrm{C}$ every few days. The humidity can change $\pm 20 \%$ due to weather or seasonal changes. In addition, the $50 \mathrm{MHz}$ laser is built in the room containing the air conditioner units used to cool the $1.3 \mathrm{GHz}$ room, generating constant background vibrations. The laser was surrounded by a box with $1 / 2$ inch thick plastic to reduce background noise. With all of these environmental variations, the laser remains well synchronized to the external clock.

The oscillator generates chirped pulses with up to $0.4 \mathrm{~nJ}$ energy, $1 \mathrm{~nm}$ spectral bandwidth, and $1.9 \mathrm{ps}$ (FWHM) pulse duration at a $50 \mathrm{MHz}$ repetition rate. The Kelly spectral sidebands, normally associated with soliton pulses, are cut off using a pair of bandpass filters to $10 \mathrm{~dB}$ below the main signal, and an average power of $10 \mathrm{~mW}$ is obtained after the filters. The time-bandwidth product of the pulses is 0.51 . The laser is synchronized to a radio-frequency (rf) clock with a relative timing jitter of less than 1 ps.

The optical pulses from the oscillator are first amplified in a preamp stage. The gain fiber of the preamp is a $10-\mathrm{cm}-$ long single-clad (SC) single-mode (SM) highly doped $\mathrm{Yb}$ fiber (Yb-406, CorActive), and is directly connected with a collimator. The gain fiber is copumped by a fiber-coupled $976 \mathrm{~nm}$ laser diode, and the pump laser beam is combined with seed light through a wavelength division multiplexer (WDM). $500 \mathrm{~mW}$ pump power yields $300 \mathrm{~mW}$ output, corresponding to a pulse energy of $6 \mathrm{~nJ}$. As the effective fiber length is less than $3 \mathrm{~cm}$, the nonlinear phase shift is $0.5 \pi$ and only slight spectral distortion is observed at the maximum output [Fig. 4(b)].

The main amplifier is a single-mode, 2- $\mathrm{m}$, singlepolarization, double-clad (DC) ytterbium-doped photonic crystal fiber with $650 \mu \mathrm{m}^{2}$ mode area and $10 \mathrm{~dB} / \mathrm{m}$ absorption at $976 \mathrm{~nm}$ (model DC-200/40-PZ-Yb-03, NKT Photonics). The fiber is coiled with a $30-\mathrm{cm}$ diameter and placed on a water-cooled aluminum plate. The amplifier is counterpumped by a fiber-coupled $976 \mathrm{~nm}$ laser diode with a power of $25 \mathrm{~W}$. Since the pump light is not perfectly coupled into the fiber, residual pump light will dissipate as heat at the ends of the fiber, potentially damaging it. To avoid this, the two ends of the gain fiber are connected with copper SMA connectors and held in copper heat sinks, water cooled to $20^{\circ} \mathrm{C}$. Rigid, fixed height optomechanical components have been used throughout the system in order to keep the laser system reliable for long-term operation.

To generate high-average electron beam currents, photocathodes with high quantum efficiency are needed, which is most easily accomplished with semiconductor cathodes 

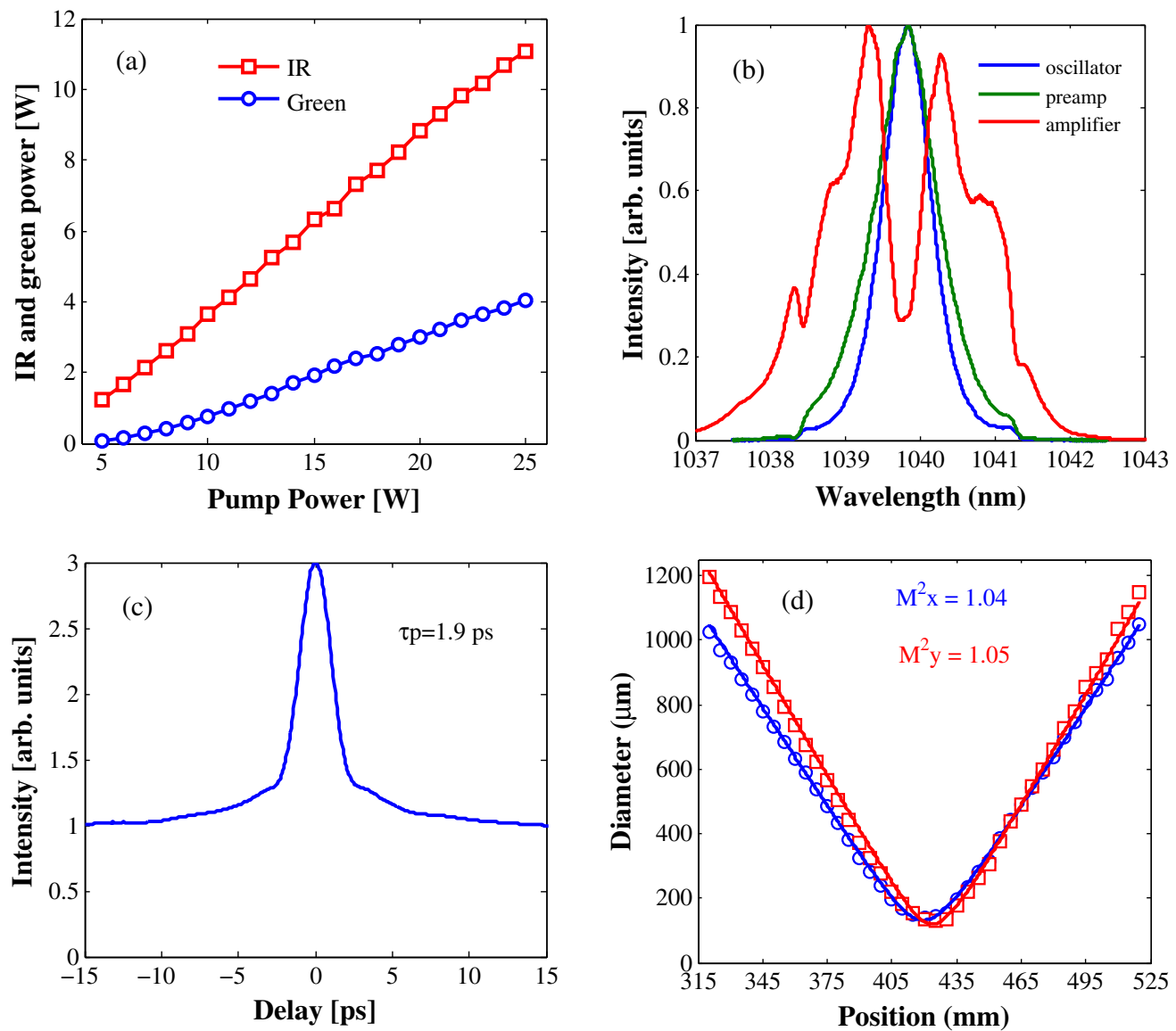

FIG. 4. The characteristics of $50 \mathrm{MHz}$ fiber amplifier: (a) IR output versus pump power; (b) optical spectrum from oscillator, preamp, and amplifier at $5 \mathrm{~W}$; (c) $\mathrm{C}$ signal of IR pulses; (d) $\mathrm{M}^{2}$ measurement results at $10 \mathrm{~W}$ IR power.

operating at visible wavelengths. To generate green light $(520 \mathrm{~nm})$, the IR beam $(1040 \mathrm{~nm})$ from the laser amplifier is focused onto a type I noncritically phase matched lithium triborate $(\mathrm{LBO})$ crystal. The $5 \times 5 \times 15-\mathrm{mm}^{3}$ crystal is placed in an oven and the temperature is varied to optimize the phase matching. The crystal has a $2 \mathrm{~nm}$ phase matching bandwidth, and the oven temperature is set at $178^{\circ} \mathrm{C}$. After the SHG crystal, a lens and two dichroic mirrors are employed to collimate the SHG beam and remove any residual $1040 \mathrm{~nm}$ light.

The characteristics of the amplified infrared (IR) pulses are shown in Fig. 4. The maximum average power is $11 \mathrm{~W}$ when $25 \mathrm{~W}$ of pump power is incident on the gain fiber. The optical-to-optical efficiency from pump to IR output power
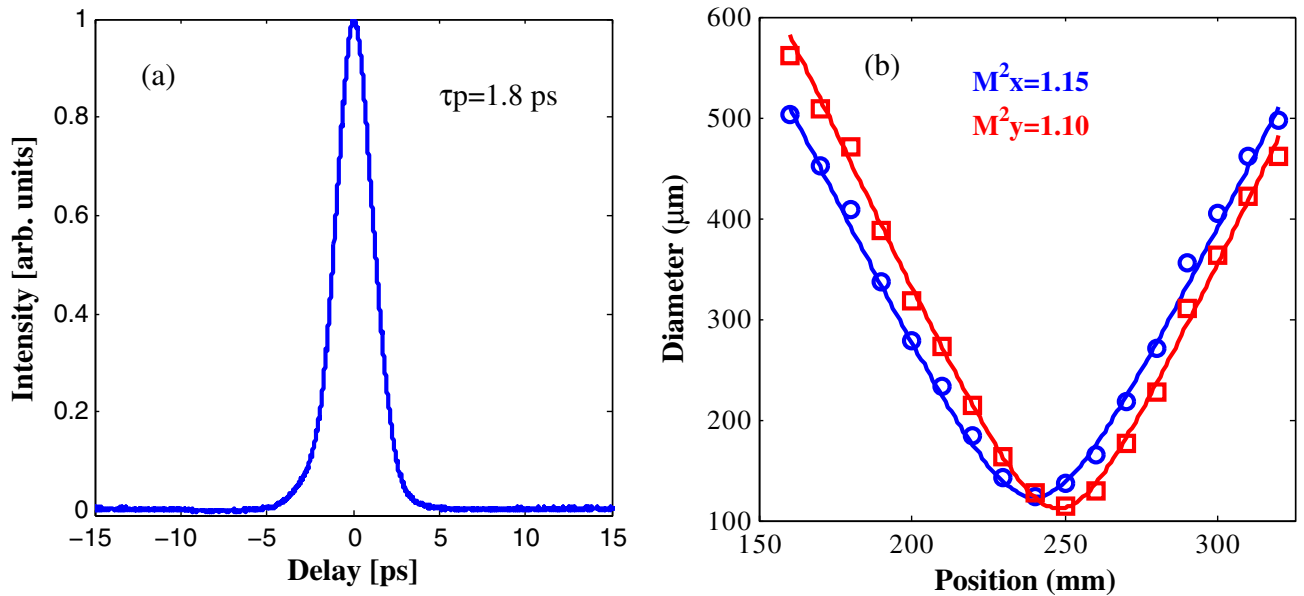

FIG. 5. (a) AC signal; (b) $\mathrm{M}^{2}$ measurements at $4 \mathrm{~W}$ average green power. 


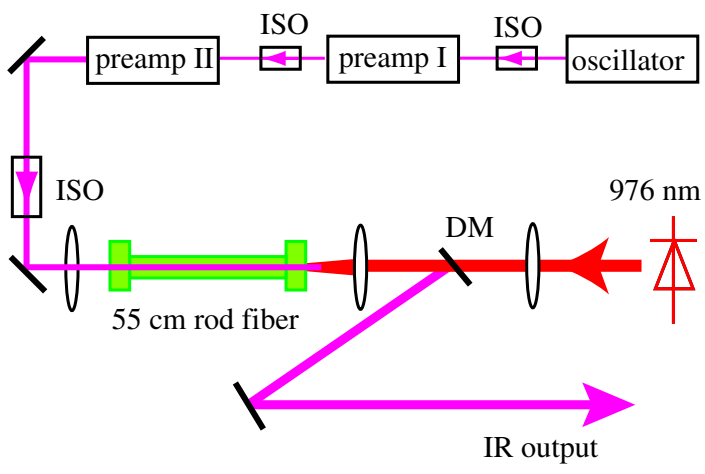

FIG. 6. Schematic of the experimental setup: a fiber oscillator, two preamps, and a main rod amplifier. ISO is optical isolator and DM dichroic mirror.

is $44 \%$. At the maximum output power, the polarization extinction ratio of the amplified pulses is above $12 \mathrm{~dB}$ and amplified spontaneous emission (ASE) is suppressed to at least $40 \mathrm{~dB}$ below the amplified pulses.

As shown in Fig. 4(b), there is some spectral distortion at $5 \mathrm{~W}$ output power, and the nonlinear phase shift accumulated during the amplification is calculated to be approximately $\pi$. At the maximum output power, there is stronger spectral distortion and the nonlinear phase shift is $2 \pi$. From a collinear autocorrelation (AC) measurement, the pulse duration is 1.9 ps [Fig. 4(c)] if a Gaussian pulse is assumed, and the time-bandwidth product is 1.0. Therefore, a pulse energy of $220 \mathrm{~nJ}$ and a peak power of $110 \mathrm{~kW}$ are achieved after the main amplifier. At the maximum power, the $\mathrm{M}^{2}$ values in horizontal and vertical directions are 1.04 and 1.05 , respectively [Fig. 4(d)], and the IR beam is near diffraction limited.

After IR pulses are steered through the PC, the average power is $8.3 \mathrm{~W}$. As shown in Fig. 4(a), a maximum power of $4 \mathrm{~W}$ green light and a conversion efficiency of $48 \%$ is achieved by the SHG. The green power is approximately linear with the IR input due to the high-peak power. The pulse duration of the green light at $4 \mathrm{~W}$ is 1.8 ps [Fig. 5(a)] if a Gaussian pulse is assumed, and the $\mathrm{M}^{2}$ values in horizontal and vertical directions are 1.10 and 1.15 [Fig. 5(b)], respectively, verifying the excellent beam quality of green light.

\section{50 MHz ROD FIBER AMPLIFIER FOR HIGHER ENERGY PER PULSE APPLICATIONS}

For certain applications, a synchronized laser with much higher energy per pulse is desired. One example is Compton scattering to measure the electron beam profile $[12,13]$. This section describes an amplifier system to reach up to $2 \mu \mathrm{J}$ per pulse at $50 \mathrm{MHz}$ repetition rates. This
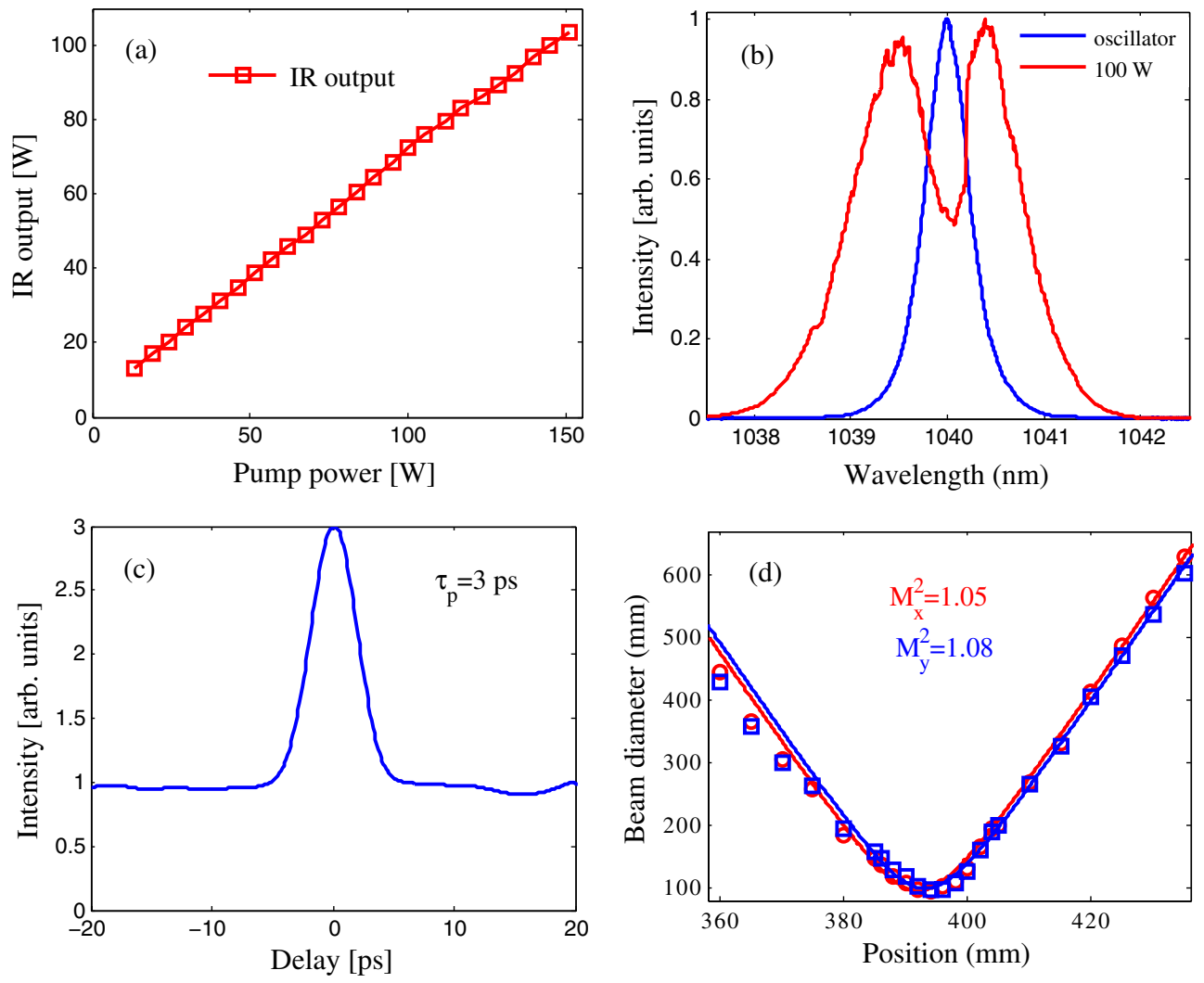

FIG. 7. The characteristics of rod fiber amplifier: (a) IR output versus pump power, (b) optical spectrum of seed light and amplified pulses at $100 \mathrm{~W}$, (d) intensity autocorrelation of $100 \mathrm{~W}$ IR output, (e) $\mathrm{M}^{2}$ values at $100 \mathrm{~W}$ IR power. 
amplifier will work for a wide range of repetition rates, but $50 \mathrm{MHz}$ is used here for consistency.

The experimental schematic of the Yb-doped rod-type fiber amplifier is depicted in Fig. 6. It consists of a fiber oscillator, two preamp stages, and one main rod amplifier [14]. Both the fiber oscillator and preamp I are similar to the previous setup in Fig. 2. In preamp II and the main amplifiers, 36-cm and 55-cm rod-type fibers with a mode field area of $4500 \mu \mathrm{m}^{2}$ (DC-285/100-PM-Yb-ROD) are used, respectively. Both rod fibers are cooled to $15^{\circ} \mathrm{C}$ using aluminum $\mathrm{V}$-groove heat sinks. Thanks to the larger mode area and shorter fiber length in the rod amplifier, we could substantially reduce the nonlinear phase shift accumulation, and boost the pulse energy by 10 times without important spectral distortion.

The oscillator is also a soliton fiber laser with a net dispersion of negative $2 \mathrm{ps}^{2}$, and it generates nearly transform-limited pulses with $0.2 \mathrm{~nJ}$ energy, $2.7 \mathrm{ps}$ duration, and $0.49 \mathrm{~nm}$ bandwidth at $1040 \mathrm{~nm}$. The time-bandwidth product is 0.37 . With the pump power of $500 \mathrm{~mW}$ and $30 \mathrm{~W}$ in the two preamp stages, the pulse energies are increased to 10 and $200 \mathrm{~nJ}$, respectively.

The amplifier output versus pump is shown in Fig. 7(a). The IR power of $100 \mathrm{~W}$ is obtained with pump power of $150 \mathrm{~W}$, corresponding to pulse energy of $2 \mu \mathrm{J}$. The slope efficiency from pump to IR output is $67 \%$. The IR output can be scaled up above $150 \mathrm{~W}$ with an improved heat sink [14]. The polarization extinction ratio of the amplified pulses is sustained more than $10 \mathrm{~dB}$ at any output power. The optical spectrum of the seed and the amplified pulses at $100 \mathrm{~W}$ is shown in Fig. 7(b). While some spectral broadening is observed, the pulse duration is $3 \mathrm{ps}$, and the $\mathrm{M}^{2}$ values in horizontal and vertical directions are 1.05 and 1.07 , respectively. Frequency doubling of amplified pulses yields $70 \mathrm{~W}$ green power with $70 \%$ efficiency. This higher power laser can be used for Compton scattering nondestructive diagnostics of the electron beam $[12,13]$.

\section{THE 1.3 GHz LASER SYSTEM AND CHARACTERIZATION}

The schematic of the $1.3 \mathrm{GHz}$ fiber amplifier is shown in Fig. 8. The amplifier is a Yb-doped fiber MOPA, and consists of a seed source, two preamplifier stages, and a main amplifier [5]. The seed source is a commercial Yb-doped fiber oscillator (PriTel), and can be operated in a variety of modes, with different pulse lengths, spectral bandwidths, and amount of chirp. In our experiment, the oscillator is harmonically mode locked to an external rf clock at $1.3 \mathrm{GHz}$, and a relative rms timing jitter of $<1 \mathrm{ps}$ is achieved. The output pulses are centered at $1040 \mathrm{~nm}$ with $15 \mathrm{pJ}$ pulse energy, $20 \mathrm{~mW}$ average power, and $2 \mathrm{~nm}$ bandwidth. Optical pulses are conveniently chirped to around 8-ps (FWHM), and can be best de-chirped to 800 -fs after amplification. The Pritel oscillator itself comes enclosed in a sealed, temperature stabilized box, set to

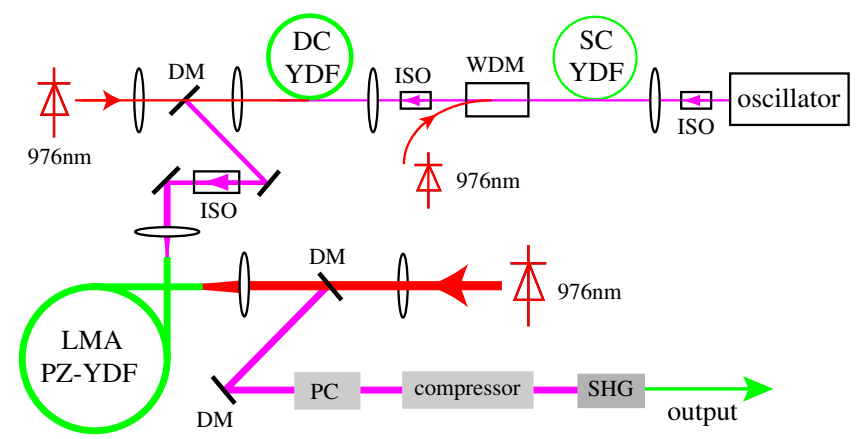

FIG. 8. Schematic of the $1300 \mathrm{MHz}$ system: LMA, large mode area; PZ-YDF, single-polarization Yb-doped fiber; WDM, wavelength division multiplexer; SC, single clad; DC, double clad; ISO, optical isolator; DM, dichroic mirror; PC, Pockel's cell; the compressor is a pair of gratings; SHG, second harmonic generation.

$35.0^{\circ} \mathrm{C}$. The laser room is temperature controlled to a stability of $0.5^{\circ} \mathrm{C}$, and has both a humidifier and dehumidifier, which are used to hold the humidity stable at $30+/-2 \%$.

The optical pulse train from the oscillator is first amplified in a single-mode gain fiber and then in a single mode polarization-maintaining (PM) double-clad (DC) Yb-doped gain fiber with a $15-\mu \mathrm{m}$ mode-field diameter (DC-135/15-PM-Yb). Both gain fibers are Yb-doped and copumped. The output power from preamp II is $3 \mathrm{~W}$ with $6 \mathrm{~W}$ of pump power. The polarization extinction ratio (PER) of the amplified pulses is $15 \mathrm{~dB}$. Since the nonlinear phase accumulation is very small, no spectral distortion is observed. Also, ASE is $30 \mathrm{~dB}$ below the main signal.

The main amplifier uses $3 \mathrm{~m}$ of the same fiber used in the $50 \mathrm{MHz}$ system. The output pulses of the main amplifier are sent through a PC and then compressed with a pair of transmission gratings with 1250 lines per $\mathrm{mm}$ (Ibsen). The compressed pulses are converted to the green through a similar SHG. Although much higher average power is generated in the $1.3 \mathrm{GHz}$ fiber amplifier, the spatial mode can be kept very stable in long-term operation. No adjustments to the amplifiers have been needed in the last 24 months of operation.

The amplifier characteristics after the compressor are shown in Fig. 9. The maximum average power is $150 \mathrm{~W}$ with $230 \mathrm{~W}$ of pump power. The slope efficiency from pump to IR output power from the amplifier is $67 \%$. However, the output is typically limited to less than $70 \mathrm{~W}$ to avoid damaging the $\mathrm{PC}$ after the amplifier. As the nonlinear phase shift accumulated by the pulse is calculated to be $0.6 \pi$ at the $150 \mathrm{~W}$ output level, almost no spectral distortion is observed, and ASE is $30 \mathrm{~dB}$ below the amplified signal. The IR output power fluctuates by about $1 \%$, attributed to a small drift of the pump laser wavelength. The PER of the amplified pulses is above $12 \mathrm{~dB}$.

After the main amplifier, the pulses are chirped with pulse duration of $8 \mathrm{ps}$, and are compressed to $1.1 \mathrm{ps}$ through 

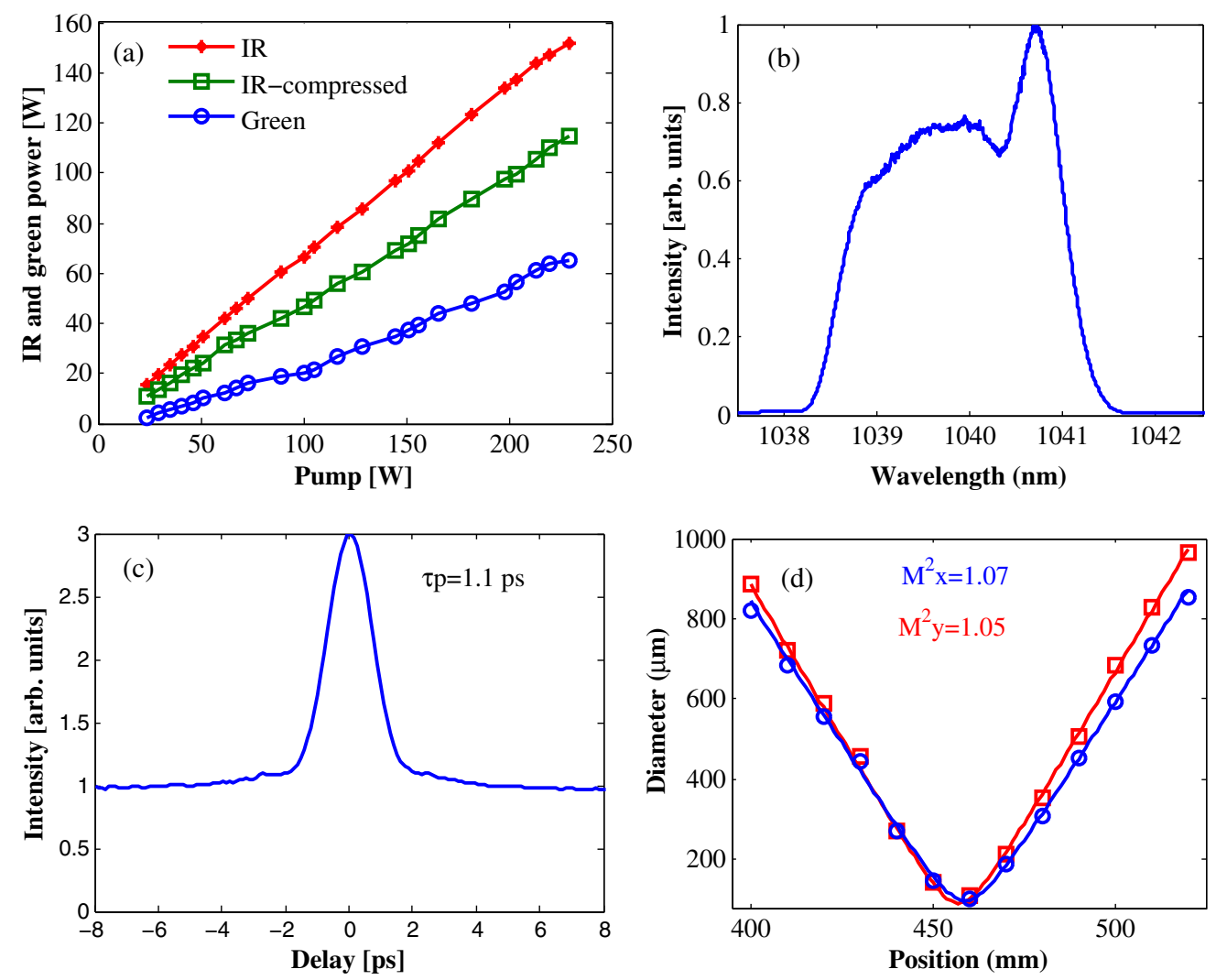

FIG. 9. (a) Compressed IR output and green power versus pump power; (b) optical spectrum of the amplified pulses at $150 \mathrm{~W}$; (c) AC signal; and (d) $\mathrm{M}^{2}$ measurement results at $85 \mathrm{~W}$ compressed IR power.

a grating pair. At the maximum pump power, $110 \mathrm{~W}$ of IR power is obtained with an overall efficiency of $74 \%$. The PER of the compressed pulses is $>20 \mathrm{~dB}$, and the $\mathrm{M}^{2}$ values in horizontal and vertical directions are less than 1.1 at any output power, so the amplified pulses are nearly diffraction-limited.

After the pulse compressor, an LBO crystal is used to carry out the frequency doubling. The conditions are the same as those used in the $50 \mathrm{MHz}$ laser system. However, it is necessary to adjust the oven temperature to compensate for heating of the LBO crystal owing to the high average power [15].

The green power is proportional to the input power, and the conversion efficiency is $60 \%$ in the high-power regime. A maximum power of $65 \mathrm{~W}$ at $520 \mathrm{~nm}$ was reached with $110 \mathrm{~W}$ of IR power. The pulse duration, measured using a non-collinear autocorrelation process, was $1 \mathrm{ps}$. The $\mathrm{M}^{2}$ values are 1.2 at low power, and increase to 1.5 in the highpower regime (see Fig. 10). The incremental $\mathrm{M}^{2}$ values can be attributed to thermally-induced distortion [15] in the
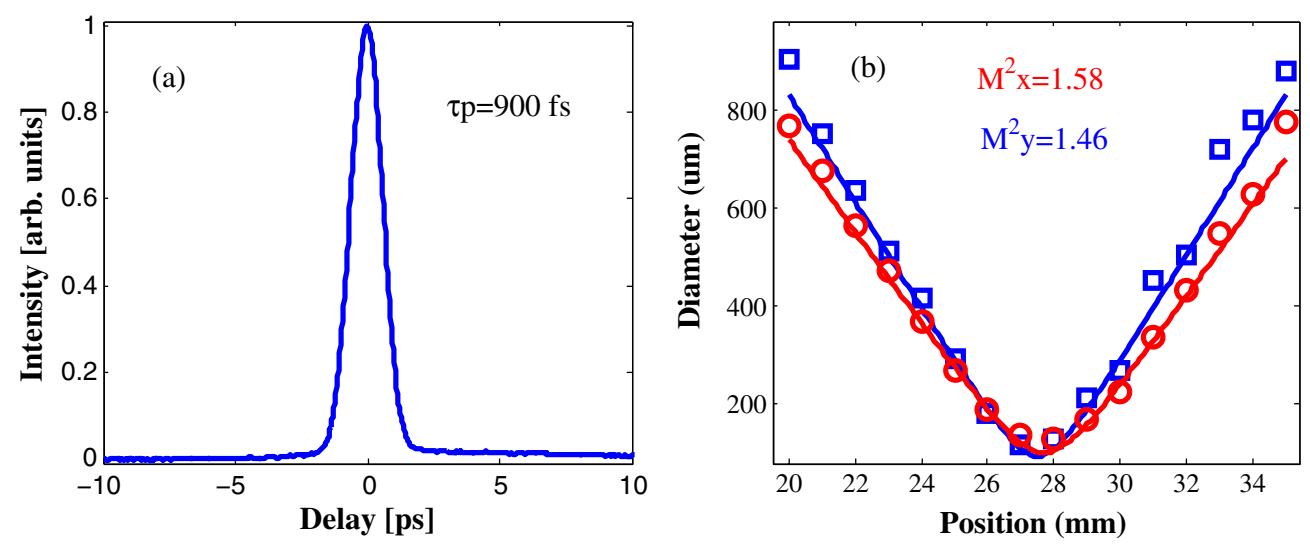

FIG. 10. (a) AC signal; and (b) $\mathrm{M}^{2}$ measurement results of green light at $50 \mathrm{~W}$. 
LBO crystal. As shown in Fig. 18, the beam profile is still excellent for driving the photocathode after the spatial mode shaping process.

\section{POCKELS CELL FOR OPTICAL PULSE PICKING}

As shown in Fig. 11, a beta barium borate (BBO) Pockels cell (Lightgate 7, Gooch \& Housego) with a driver (PCD3d, BME) is used to reduce the duty factor of optical pulses. This allows one to reduce the average power of the electron beam without changing the parameters of the individual pulses reaching the photocathode. There are two modes of operation, which we refer to as "CW" and "Pulsed", selected with an insertable half-wave plate (HWP) after the PC. For "CW" operation, the HWP is removed and the $\mathrm{PC}$ is not powered. This allows all pulses to proceed to the SHG crystal. In "Pulsed" mode, the HWP is inserted. When the PC is off, the pulses will pass through the subsequent polarizer and into a beam dump. When it is on, the polarization is rotated by 90 degrees, allowing the pulse to continue to the SHG crystal. This PC is limited to duty factors below $5 \%$, with rate smaller than $5 \mathrm{kHz}$ and duration from $80 \mathrm{~ns}$ to $10 \mu \mathrm{s}$. BBO crystal is used as it is

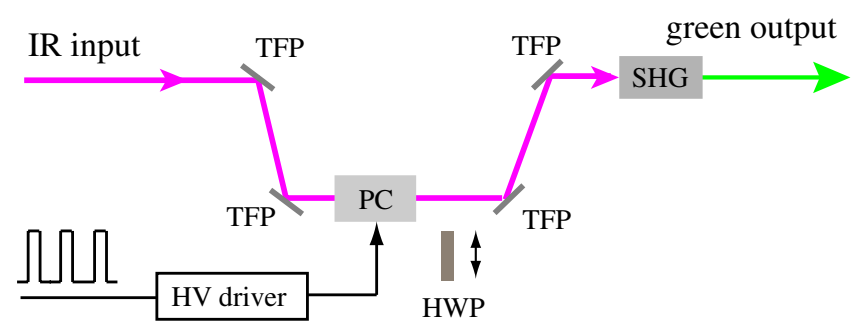

FIG. 11. PC, TFP, and SHG are combined to achieve high extinction ratio pulse picker. PC, Pockel's cell; TFP, thin film polarizer; HWP, half-wave plate; SHG, second harmonic generation.

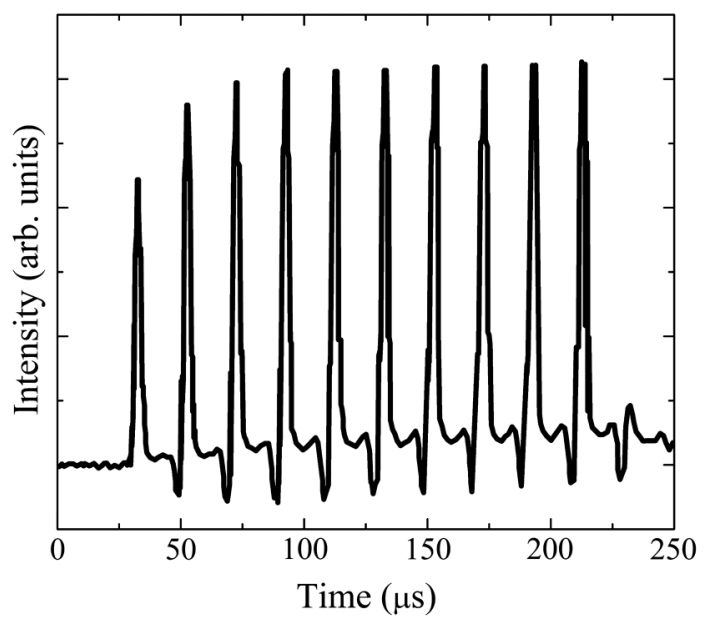

FIG. 12. Output pulse train from a BBO PC. There is no ringing, but the first few pulses have lower energy. known to have fast rise and fall times $(<5 \mathrm{nsec})$ with minimal ringing. This ensures that each pulse in the pulse train with have nearly the same energy, which is important for electron beam characterization (see Fig. 12).

To improve the on/off extinction ratio, two pairs of thin film polarizers (TFPs) are installed both in front of and behind the PC. The TFPs have the advantage of a high damage threshold and good extinction ratio at the Brewster angle (56 degrees). The on/off extinction ratio of the optical pulses was optimized to exceed 1000:1 in the IR. In the subsequent $520 \mathrm{~nm}$ beam, the ratio is better than $10^{6}$ to 1 as the green power is quadratically dependent on the IR power. This is why it is critically important to place the SHG after the PC. Otherwise, the extinction will be poor, resulting in longitudinal electron beam halo.

\section{GREEN LIGHT INTENSITY CONTROL AND ELECTRON BEAM CURRENT STABILIZATION}

After the fiber amplifier and the pulse compressor, the intensity fluctuation of the IR power is less than $1 \%$, and the green power behind the SHG crystal exhibits similar fluctuation. As the laser beam will be transported $30 \mathrm{~m}$ followed by a small aperture (for transverse shaping), poor pointing stability translates to poor power stability after clipping on this aperture. All these factors may add up to 5-10\% fluctuations in the green power when the laser pulses reach the photocathode. As a result, unwanted current variation and frequency noise will occur in the electron beam.

To correct for these variations, a digital fast-feedback system was implemented to reduce the current fluctuations to $<0.5 \%$ [16]. The electron beam current is sampled by summing the four pickups from a beam position monitor after the electron gun. The signal is referenced to a DC current to produce an error signal. The laser power after the SHG is modulated using another PC (Conoptics 350-105), driven with a high voltage amplifier (TEK 2100HF). A digital PID control loop was implemented to keep the electron beam current constant by varying the signal to the modulator.

The PC has a bandwidth of $\sim 1 \mathrm{MHz}$, so it can also be used as a fast laser shutter for machine protection. If the accelerator control system detects a fault that can cause beam loss, it will send a signal to block the laser beam and thus terminate the electron beam and protect the accelerator from damage. With a $1 \mathrm{MHz}$ bandwidth, the laser can be shuttered within $\sim 1$ us after receiving a shutdown signal.

\section{TEMPORAL SHAPING OF LASER PULSE}

The approach to longitudinal pulse shaping is to make use of a stack of birefringent crystals [6-8]. The horizontally-polarized optical pulses are separated into sixteen pulses through a set of four $\alpha$-cut $\mathrm{YVO}_{4}$ crystals. The fourcrystal stack is placed in order of decreasing length (15.1, 


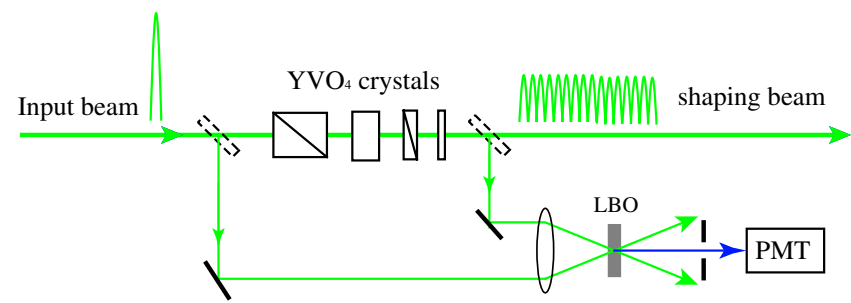

FIG. 13. Laser beam shaping and cross-correlation measurement. Four crystals are alternatively oriented at 45 degrees and 0 degrees to achieve the longitudinal shaping and a cross correlator is used to measure the pulse duration.

$7.55,3.775$, and $1.887 \mathrm{~mm}$ ) and are alternatively oriented at 45 and 0 degrees, as shown in Fig. 13. The four-crystal stack can separate each input pulse into sixteen pulses with alternative polarization, and lead to a temporal profile with $\sim 30$ ps pulse duration since the group delay difference between the fast and slow optical axis is $1.05 \mathrm{ps} / \mathrm{mm}$. The rise and fall time of the laser shaping beam is determined by the primary input pulse width. The temporal profile of laser shaping beam is measured through a cross-correlator with reference to the input pulses, and measurements of two polarization components of the shaped pulses are used to reconstruct the temporal beam profile. Each surface of the crystal can generate 1-2\% loss and the total loss through the crystal stacks is about $10 \%$.

Figures 14(a) and 14(b) show the cross-correlation measurements of the shaping laser pulses and the electron longitudinal beam profiles from the $50 \mathrm{MHz}$ and $1.3 \mathrm{GHz}$ laser systems, respectively. Optical pulses with the duration of $30 \mathrm{ps}$ (a rms of $8 \mathrm{ps}$ ) are generated with this longitudinal pulse shaping configuration, and the efficient temporal shaping of electron distributions produced accordingly. Due to the shorter duration with a $1.3 \mathrm{GHz}$ laser system, the longitudinal profile produces a higher ripple and sharper rise and fall than the one from the $50 \mathrm{MHz}$ laser system. In contrast to the previous experiments [6-8], we have intentionally used a shorter duration in the input pulses and thus produced a small ripple in the shaping pulses instead of truly "flattop" pulses. For truly flattop pulses, the pulses would need to be so close that interference effects become important for the pulse profile. Thus, slight changes in temperature (thus changing crystal length or index) or other factors could have a dramatic impact on the profile, hindering long-term stability. Though there is some modulation of the laser profiles, the electron temporal bunch profiles have almost been smeared out, possibly due to space charge near the gun. In the operation (not shown), the electron bunch length is typically compressed to $<3$ ps rms through an rf bunching cavity.

Detailed simulations show that the final beam emittance is not affected by the longitudinal profile modulations of the laser if they remain within 50\% so that both temporal profiles shown in Fig. 14 are acceptable to generate bunches with nearly identical low emittance [17].

\section{LASER BEAM TRANSPORT}

While the $1.3 \mathrm{GHz}$ laser room is climate controlled, the laser transport pipes and optical table near the electron gun are not and, by virtue of being near a loading dock open to the building exterior, can have large temperature and humidity variations. This prevents us from using humidity-sensitive optical components near the electron gun, which has not been a problem otherwise.

As shown in Fig. 15, the laser beam is transported from the laser room to the photoemission gun through a long $1: 1$ imaging system. It is first lifted to a pipe, travels $22 \mathrm{~m}$, and finally descends to an optical table for further transverse shaping and control. Between the laser room and injector, the pipeline is sealed to prevent air turbulence (caused by a temperature differential). All the mirrors are chosen to be highly reflective $(\mathrm{R}>99.9)$ at $520 \mathrm{~nm}$ (custom from Laytec). The target is an aperture, which has an adjustable diameter from $0.2-7.0 \mathrm{~mm}$. In typical operation it is fixed at $2.0 \mathrm{~mm}$ diameter. The goal is to image a Gaussian-shaped laser beam on this aperture, truncating the edges of the beam sharply. Thus, it is important to keep the beam's position on this aperture stable to about 10 micron rms.
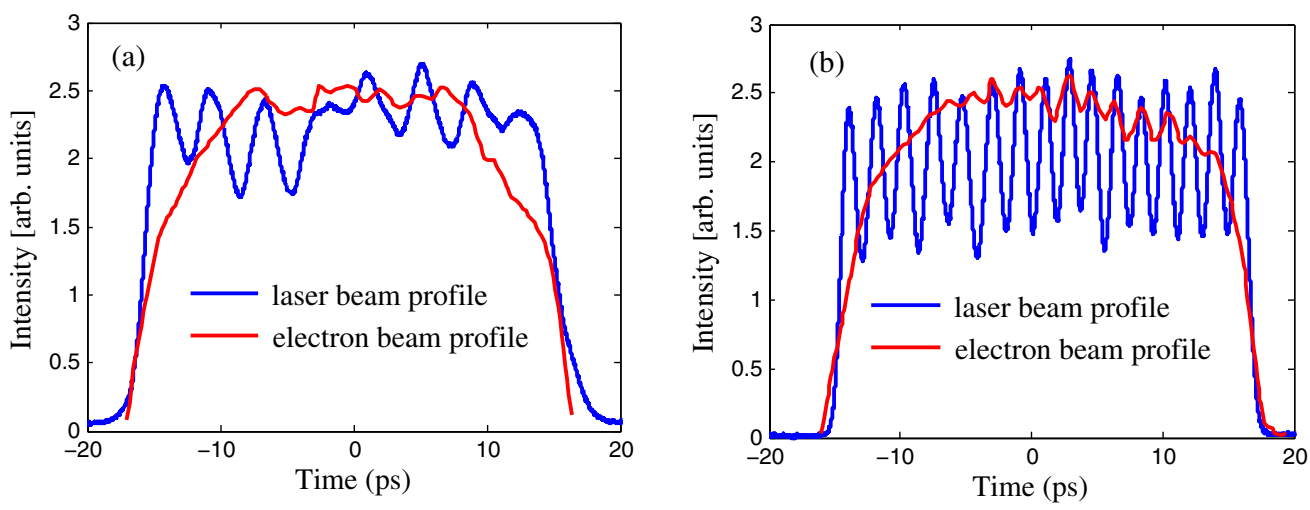

FIG. 14. (a) The laser and electron bunch profile for the $50 \mathrm{MHz}$ laser and (b) for the $1.3 \mathrm{GHz}$ laser. 
Laser beam transport tunnel

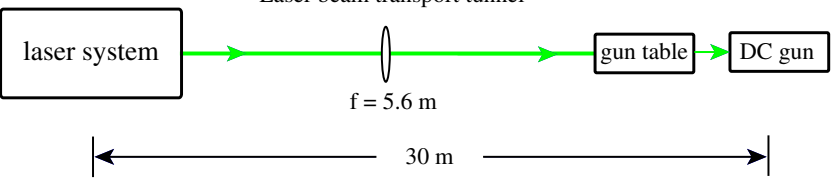

FIG. 15. The laser beam transport system. Highly reflective mirrors, a telescope and rigid mounts are used to transport optical pulses from the laser room to the optical table near the DC gun.

Imaging 1:1 from the point behind the SHG crystal to the target provides the best performance.

To conduct the laser beam transport, a system of lenses is used to image the plane at the SHG crystal onto the spatial shaping aperture near the electron gun. For the $1.3 \mathrm{GHz}$ laser, a $1 \mathrm{~m}$ lens is used to image the SHG crystal onto the motorized "flip mirror" that selects which laser beam (50 $\mathrm{MHz}$ or $1.3 \mathrm{GHz}$ ) is sent to the gun. For the $50 \mathrm{MHz}$ laser, two lenses are used since it has a longer path length to the same flip mirror. This image plane is then imaged onto the shaping aperture by the gun with a single $\mathrm{f}=5.6 \mathrm{~m}$ lens placed halfway down the remaining $22 \mathrm{~m}$ of pipe. The imaging was fine-tuned by adjusting the position of the first $\mathrm{f}=1 \mathrm{~m}$ lens for both lasers.

Initially, the primary sources of pointing instability were the lack of rigid optical mounts. Without those, realignment of the entire path was needed on a weekly basis. After swapping to rigid mounts, long-term stability improved such that realignment has never been needed, except when required due to a change in the laser system. Nevertheless, there were still somewhat dramatic short-term instabilities. Fast drifts on the aperture on the order of $\mathrm{mm}$ were possible, and distortions in the shape of the mode were common.

These problems were alleviated with two additional changes. First, a noticeable air current could be felt traveling down our transport pipes, due to the temperature changes in the two rooms. Adding a window to the pipe at

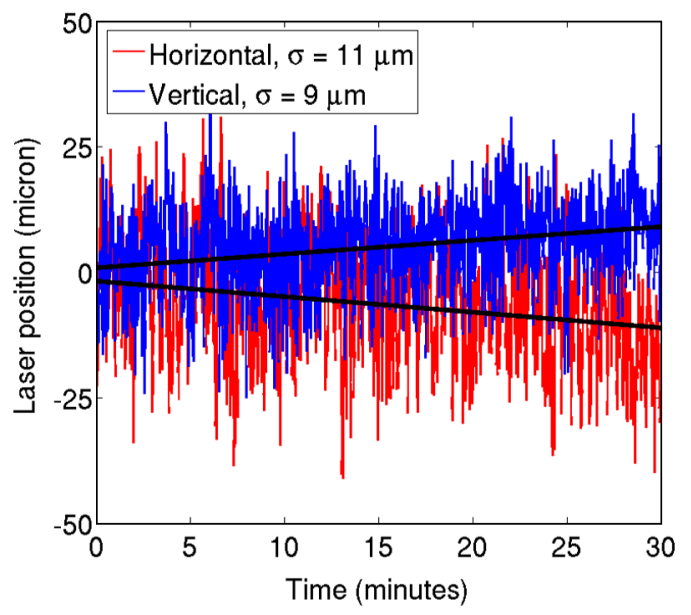

FIG. 16. Laser position stability versus the time. the entrance improved the stability significantly, and removed most of the distortions in the mode at low power. Second, we moved the laser beam dumps on the optical table far away from any path of the laser. During high power operation they would heat up, causing turbulence in the air around them. After moving these, distortions in the laser mode disappeared at high power, and the position stability improved dramatically.

Currently, the fast position jitter on the aperture is around 10 micron rms, with a slow drift of around 20 micron/hour (Fig. 16). No active position stabilization has been needed, and the slow drift is manually corrected as needed, with motorized mirrors near the electron gun.

\section{SPATIAL SHAPING OF LASER BEAM}

The final emittance of the electron beam is closely tied to the precise shape of the incident laser beam. The ideal laser profile to produce the best emittance is a complex shape, which depends on many parameters. Importantly, the emittance does not suffer greatly when using a radially truncated Gaussian profile. Optimal values of both the size of the Gaussian and the cutoff radius depend on the parameters such as the bunch charge and the temporal laser profile. So, the goal of the system was to make these two parameters adjustable.

As shown in Fig. 17, to achieve this, the beam size of transported laser pulses is first adjusted between 0.5 to $5 \mathrm{~mm}$ by using a three lens telescope ( $\mathrm{f}=10,-2,50 \mathrm{~cm}$, respectively). The lenses were chosen to give a tunable magnification from $1-10 \mathrm{X}$ by translating the middle lens over a $25 \mathrm{~mm}$ range, while maintaining a nearly collimated output beam. The beam passes through an aperture with size selectable from a set that ranges from $0.2-7.0 \mathrm{~mm}$. The pinholes were premounted and aligned on a set of magnetic bases, for fast swapping. This aperture is then $1: 1$ imaged using a single $\mathrm{f}=75 \mathrm{~cm}$ lens onto the photocathode surface (Fig. 18), with $0.5 \%$ pickoff mirrors behind the pinhole and the imaging lens used to monitor the spatial modes. Figure 18 shows an example profile created with this system, measured using a $0.5 \%$ pickoff mirror during high power operation.

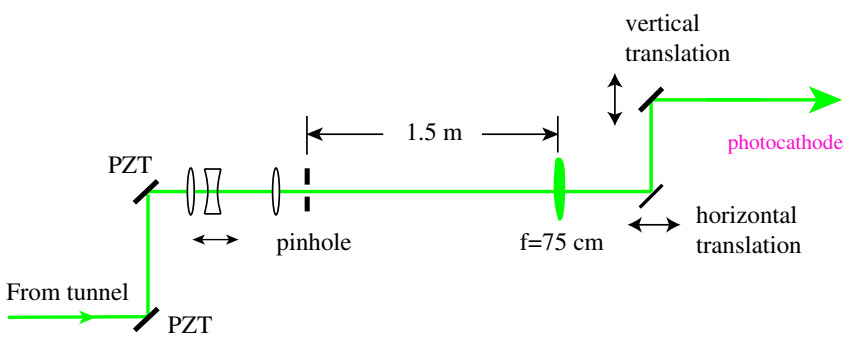

FIG. 17. Layout of the optical table near the electron gun. Spatial shaping and positioning is performed here using an adjustable telescope, pinhole, motorized stages, and a $1: 1$ imaging system. 

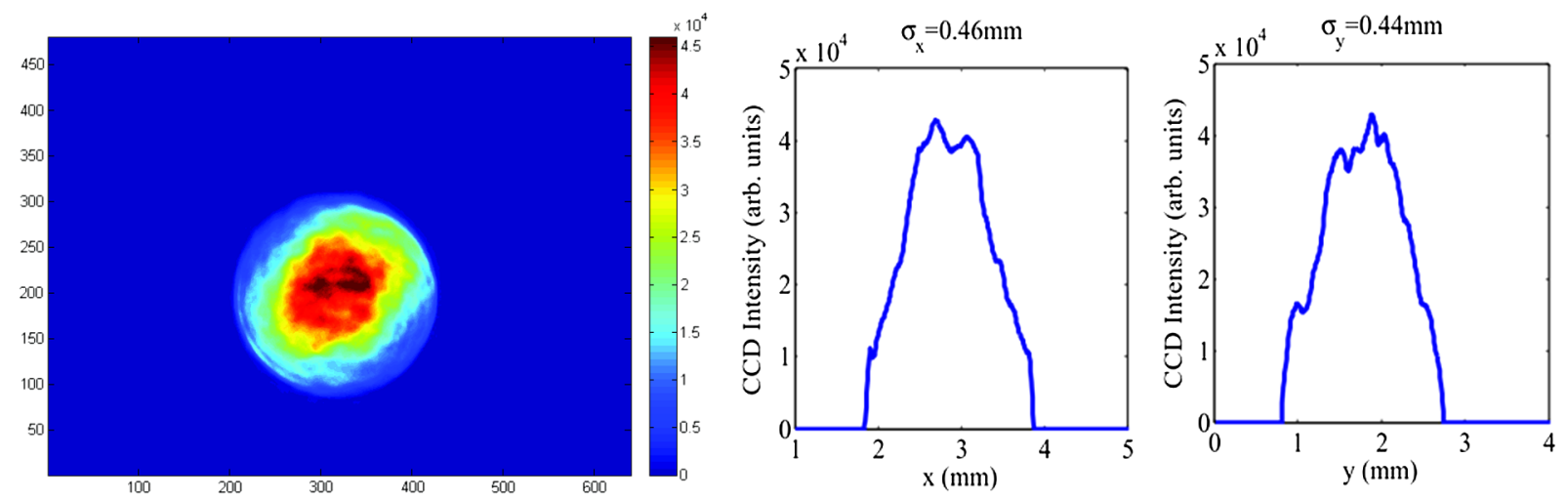

FIG. 18. Laser profile in the high-power operation (left), and the laser profile measured during high power operation (right).

To control the position of the laser both on the photocathode and also on the in-vacuum mirrors within the gun, four controls are used. Two motorized stages (Fig. 17) are used to translate the final two mirrors before the gun, vertically and horizontally, causing the laser spot to also translate. In addition, the final mirror has motorized tip/tilt control. In this way, the desired spot on the cathode can be hit, while still maintaining good alignment in and out of the gun.

Finally, there is an optional motorized mirror (not shown) that can intercept the beam right before it enters the gun. This beam is then directed either towards a thermal high power meter, to reliably tell the laser power without needing a scale factor, or it can be directed onto a CCD positioned at the same distance away as the cathode for reliable laser positioning and imaging onto the cathode.

The total loss of our transport system, defined as the ratio of laser power at the cathode to laser power at the SHG crystal, is very large. The electro-optic modulator used for power stability has $20 \%$ loss, the longitudinal shaping crystals have $10 \%$ loss, clipping the laser beam on the pinhole aperture can have huge losses depending on the size of the aperture, but is typically $30 \%$ or more. The sum of all other intervening optics has an additional $10 \%$ loss. Altogether, beam transport will typically lose $60 \%$ or more of the power initially in the laser beam. For example, at our highest currently demonstrated electron beam current of $75 \mathrm{~mA}$, up to 5 watts of laser power was available for the photocathode, though nearly $20 \mathrm{~W}$ were being originally produced.

\section{APPLICATIONS OF HIGH-POWER LASERS FOR LOW EMITTANCE MEASUREMENT AND HIGH AVERAGE CURRENT ELECTRON BEAM}

One of the main goals of the Cornell ERL injector is to achieve a low-emittance electron beam after the injector acceleration section. The $50 \mathrm{MHz}$ laser system was used to perform these measurements. By carefully controlling the spatial and temporal profiles of the laser beam, optimized emittances at both 20 and $80 \mathrm{pC}$ have been obtained [9]. If subsequently accelerated to $5 \mathrm{GeV}$, the beam's brightness would be 20 times larger than previously demonstrated [9].

A high-power, high-average current electron beam is a second major goal with the Cornell injector. Using the 1.3 $\mathrm{GHz}$ laser, the injector has demonstrated up to $75 \mathrm{~mA}$ at $4 \mathrm{MeV}$, for a short period and $65 \mathrm{~mA}$ for an extended duration, a record high average current electron beam $[18,19]$. Though up to $65 \mathrm{~W}$ at $520 \mathrm{~nm}$ have been obtained in the high-power laser system, an average power of $20 \mathrm{~W}$ is sufficient for this operation, including overhead for laser shaping, intensity control and optical transport losses.

After the initial experiment, the machine could be operated at $65 \mathrm{~mA}$ beam current for many hours, and the $\mathrm{QE}$ of the photocathode was measured to be about 6\%, degrading slowly with a 65 hour (1/e) lifetime [19]. With $>50 \mathrm{~W}$ of available laser power, which would be sufficient for more than 5 days of continuous operation before a new photocathode is required.

\section{CONCLUSION}

In summary, we have reported on the high-power fiber laser systems used for the Cornell ERL photoinjector prototype. High-power fiber laser amplifiers, spatiotemporal beam shaping, and a number of controls have been described and used to achieve low emittance and high average current. Although the high-power laser system is specifically designed for the Cornell photoinjector, the techniques demonstrated are suitable wherever a highpower, picosecond laser is required.

\section{ACKNOWLEDGMENTS}

We would like to thank John Dobbins and Florian Loehl for electronics control and Karl Smolenski and Tobey Moore for mechanical engineering support. The ERL photoinjector program at Cornell University is supported by the NSF, under Grant No. DMR-0807731. 
[1] J. L. Abelleira J. Phys. G 39, 075001 (2012).

[2] V. N. Litvinenko and Y. S. Derbenev, Phys. Rev. Lett. 102, 114801 (2009).

[3] D. H. Bilderback, J.D. Brock, D.S. Dale, K. D. Finkelstein, M. A. Pfeifer, and S. M. Gruner, New J. Phys. 12, 035011 (2010).

[4] I. V. Bazarov et al., "Technical Report," Cornell University, 2012, http://www.classe.cornell.edu/ERL/.

[5] Z. Zhao, B. M. Dunham, I. Bazarov, and F. W. Wise, Opt. Express 20, 4850 (2012).

[6] S. Zhou, D. Ouzounov, F. Wise, I. Bazarov, B. Dunham, and C. Sinclair, Appl. Opt. 46, 8488 (2007).

[7] I. V. Bazarov, D. G. Ouzounov, B. M. Dunham, S. A. Belomestnykh, Y. Li, X. Liu, R. E. Meller, J. Sikora, and C. K. Sinclair, Phys. Rev. ST Accel. Beams 11, 040702 (2008).

[8] A. K. Sharma, T. Tsang, and T. Rao, Phys. Rev. ST Accel. Beams 12, 033501 (2009).

[9] C. Gulliford, A. Bartnik, I. Bazarov, L. Cultrera, J. Dobbins, B. Dunham, F. Gonzalez, G. Hoffstaetter, S. Karkare, H. Lee, H. Li, Y. Li, X. Liu, J. Maxson, C. Nguyen, K. Smolenski, and Z. Zhao, Phys. Rev. ST Accel. Beams 16, 073401 (2013).
[10] D. G. Ouzounov, I. V. Bazarov, B. Dunham, C. Sinclair, S. Zhou, and F. W. Wise, in Proceedings of the 22nd Particle Accelerator Conference, Albuquerque, New Mexico (IEEE, New York, 2007), pp. 530-532.

[11] K. Tamura, L. E. Nelson, H. A. Haus, and E. P. Ippen, Appl. Phys. Lett. 64, 149 (1994).

[12] G. A. Krafft and G. Priebe, Rev. Accel. Sci. Techol. 03, 147 (2010).

[13] W. P. Leemans, R. W. Schoenlein, P. Volfbeyn, A. H. Chin, T. E. Glover, P. Balling, M. Zolotorev, K. J. Kim, S. Chattopadhyay, and C. V. Shank, Phys. Rev. Lett. 77, 4182 (1996).

[14] Z. Zhao, B. M. Dunham, and F. W. Wise, J. Opt. Soc. Am. B 31, 33 (2014).

[15] K.-H. Hong, C.-J. Lai, A. Siddiqui, and F. X. Kärtner, Opt. Express 17, 16911 (2009).

[16] F. Loehl, and P. Szypryt, in Proceedings of the 2nd International Particle Accelerator Conference, San Sebastián, Spain (EPS-AG, Spain, 2011), pp. 523-526.

[17] J. Maxson (private communication).

[18] B. Dunham et al., Appl. Phys. Lett. 102, 034105 (2013).

[19] L. Cultrera, S. Karkare, B. Lillard, A. Bartnik, I. Bazarov, B. Dunham, W. Schaff, and K. Smolenski, Appl. Phys. Lett. 103, 103504 (2013). 